\title{
Adaptive control of the WEDM process using a self-tuning fuzzy logic algorithm with grey prediction
}

\author{
W. M. Lee • Y. S. Liao
}

Received: 24 May 2005 / Accepted: 18 March 2006 / Published online: 8 June 2006

(C) Springer-Verlag London Limited 2006

\begin{abstract}
A control system to improve the efficiency of machining a workpiece with varying thickness in the wire electrical discharge machining (WEDM) process is proposed. The abnormal ratio $R_{a b}$ defined by the proportion of abnormal sparks in a sampling period is taken as the controlled variable. It is allowed to reduce temporarily as the cutting thickness is changing. A gain self-tuning fuzzy control algorithm is used so that the transient situation as the cutting thickness is suddenly increasing can be suppressed immediately, and a stable performance can be achieved. In addition, the grey predictor is adopted to compensate the time-delayed $R_{a b}$ caused by the low-pass filter data processing. Experiments reveal that there is a slight variance in the optimal reference of $R_{a b}$ when the cutting thickness is larger than $20 \mathrm{~mm}$, and its value is set to $55 \%$ in these cases. Three cases were tested: the constant machining parameters, the constant $R_{a b}$ and the proposed adaptive $R_{a b}$. The results show that the cutting speed can be obviously improved by the proposed control strategy.
\end{abstract}

Keywords WEDM - Machining efficiency ·

Abnormal ratio - Grey predictor · Self-tuning fuzzy controller

W. M. Lee $(\square)$

Department of Mechanical Engineering,

China Institute of Technology,

245, Sec.3, Academia Road, Nankang,

Taipei, Taiwan 11529, Republic of China

e-mail: wmlee@cc.chit.edu.tw

Y. S. Liao

Department of Mechanical Engineering,

National Taiwan University,

1, Sec. 4, Roosevelt Road,

Taipei, Taiwan 10764, Republic of China

e-mail: liaoys@ntu.edu.tw

\section{Introduction}

Wire electrical discharge machining (WEDM), one of the nontraditional manufacturing processes [1], is widely used in mold and die industries in recent years. Instead of direct contact, the workpiece is thermally eroded ahead of the traveling wire by spark discharges. Accordingly, materials with high strength and high hardness can be more easily machined than before. However, each spark discharge removes only a minute amount of material. Hence it always takes a long time to complete an entire machining run. In rough machining, any improvement in cutting speed can significantly reduce the entire production time. Accordingly, the key technique is how to increase the cutting speed. In this paper, our efforts will primarily focus on improving the cutting speed in WEDM process, especially in machining the workpiece with varying thickness.

Improving machining efficiency as well as avoiding wire rupture in the WEDM process has been studied in the past decades. Kinoshita et al. [2] found that the percentage of micro short circuit is in connection with the wire breakage. Hence they suggested to control the work feedrate by holding this percentage at a predefined constraint level. Tanimura and Heuvelman [3] found that wire breaking is related to the unusual increment of the short-circuit pulses. By detecting such type of pulse, the WEDM process is controlled by adjusting the inductance of its pulse generator. Huang et al. [4] designed an on-line identification algorithm to estimate instantaneous erosion rate and discharge gap. According to the estimated gap width, the feedrate can be controlled steadily when the workpieces vary in thickness. Zhang et al. [5] proposed to model the machining process and then the feedrate was controlled based on the developed model and the discharge conditions. 
Rajurkar and Wang [6] developed an on-line identification algorithm to estimate the workpiece height. Based on a linear model for WEDM process, an integral controller was adopted to adjust the pulse off-time to keep the spark frequency at optimal level. The optimal reference was determined experimentally according to the estimated thickness. Experimental results show that the proposed control system can successfully control the spark frequency at the optimal value without the risk of wire rupture, when cutting a workpiece with variable heights. However, it is known that the WEDM process is a complicated dynamic system and is difficult to be mathematically modeled. Such model-based control strategy $[5,6]$ is inapplicable to the wide range variation of machining condition in WEDM. Another common drawback mentioned above is that only one machining parameter was tuned in their control systems, for example feedrate in $[2,4,5]$ and pulse offtime in [6]. To augment the range of the working condition, Yan and Liao [7] proposed a multi-region fuzzy controller to simultaneously tune the federate and pulse off-time in their control system. Liao and Woo [8] developed a DSPbased on-line pulse monitoring system, and both short ratio and discharging energy were taken as controlled variables. Experimental results show that a high machining efficiency as well as system stability can be achieved in machining workpiece of constant thickness. The condition where there is a change of workpiece thickness is not taken into account. Lee and Liao [9] performed the wire rupture prevention in their proposed control system, taking the constant reference based on the worst case and using the servo voltage and arc-off-time as tuned parameters. Although the problem of wire rupture can be successively solved in many practical situations, the resulting machining efficiency and tracking performance are not good enough in machining a workpiece with varying thickness.

Machining a workpiece with varying thickness often leads to crucial cutting condition due to geometrical discontinuity. Thus the simple FLC with constant scaling factors, like gains in traditional control system, is inherently incapable of dealing with the transient and steady-state machining condition at the same time. Specifically, as the cutting thickness increases, especially under the high-speed machining condition, the gain should be large enough to suppress the transient response and avoid wire breaking. But this high gain will be apt to produce fluctuated control actions when the cutting thickness remains unchanged. Consequently, a gain self-tuning fuzzy controller was adopted to allow the desired gain modification when required.

According to the previous studies of one of the present authors [10], the abnormal ratio $R_{a b}$ was employed to evaluate the machining speed as well as to provide early warnings for wire rupture. The on-line measured $R_{a b}$ signal has extreme fluctuation due to the stochastic character of the WEDM process. Instead of being directly sent to the controller, it is usually pre-processed by the low-pass filter. But this often causes a time-delayed problem, especially when the low-pass filter has a low cut-off frequency. In order to immediately detect the symptom of impending wire rupture, i.e., the dramatic increment of the $R_{a b}$, and let controller take instant control actions, the grey predictor [11] was used to compensate the delayed filtered signal.

In rough machining, the optimal reference for the $R_{a b}$, $\left(R_{a b}\right)_{r e f}$, in the control system is the level at which the cutting speed is rather fast but allows no wire breaking. From experimental results on $\left(R_{a b}\right)_{r e f}$, the variation of its optimal setting is very small when the cutting thickness is larger than $20 \mathrm{~mm}$. Consequently, the optimal $\left(R_{a b}\right)_{\text {ref }}$ can be set to a constant value when the minimum thickness of the workpiece is larger than $20 \mathrm{~mm}$. To improve the cutting speed of machining a workpiece with changeable thickness under the fast machining condition, a strategy is proposed that the constant optimal $\left(R_{a b}\right)_{r e f}$ can be temporarily reduced to cope with the dramatically transient situation as the cutting thickness is suddenly increasing. Finally, the simultaneous adjustment of the arc off-time and the feedrate to keep the $R_{a b}$ at an optimal level was implemented to make the WEDM process more efficient.

\section{Experimental setup and electrical discharge monitoring system}

\subsection{Experimental setup}

A flushing-type WEDM machine developed by ITRI (Industrial Technology Research Institute) and CHMER Company, Taiwan, was used as our experimental machine. This machine comprises four major subsystems: an isoenergy pulse generator, a dielectric regeneration system, a motion-control system and a semiautomatic wire threading system. The adjustable machining parameters of this machine are listed in Table 1 . They can be tuned by the operator, or possibly by the control system, to improve machining efficiency or accuracy. These machining parameters include on-time, off-time, arc on-time, arc off-time, feedrate, wire speed, wire tension, and flushing pressure. Two types of pulse, normal pulse and abnormal pulse, are discriminated in our sparking monitoring system, which will be described in the next section. When an abnormal pulse is detected, arc on-time and arc off-time are generated in the pulse generator to represent the associated pulse ontime and off-time, otherwise those are called on-time and off-time if a normal pulse is detected. In practice, the setting of arc on-time is smaller than that of on-time. Some of these machining parameters must be predefined and 
Table 1 Adjustable machining parameters of WEDM machine

\begin{tabular}{ll}
\hline Item & Range \\
\hline On-time & $1 \sim 9$ steps (unit: $0.1 \mu \mathrm{s})$ \\
Off-time & $8 \sim 50$ steps(unit: $1 \mu \mathrm{s})$ \\
Arc on-time & $1 \sim 5$ steps(unit: $0.1 \mu \mathrm{s})$ \\
Arc off-time & $9 \sim 50 \mathrm{steps}($ unit: $1 \mu \mathrm{s})$ \\
Servo reference voltage & $30 \sim 70 \mathrm{~V}$ \\
Feedrate & $\mathrm{mm} / \mathrm{min}$ \\
Wire feed & $1 \sim 16 \mathrm{~m} / \mathrm{min}(15 \mathrm{step})$ \\
Wire tension & $300 \sim 1,600 \mathrm{gf}(15 \mathrm{step})$ \\
Flushing & $0 \sim 20 \mathrm{~kg} / \mathrm{cm}^{2}(7 \mathrm{step})$ \\
\hline
\end{tabular}

remain constant during the machining process because they influence the quality of the final product. For example, the surface roughness of the product is related to the spark energy of each discharge which depends on the set value of on-time or arc on-time. If their values are altered, the surface finish will be degraded. But some of the other machining parameters may be tuned without significantly affecting the surface finish and accuracy features during the machining process, such as off-time and feedrate. In addition, from both the results of influences of the machining parameters on machining characteristics [12, 13] and the viewpoint of the controllability, we selected the arc off-time and feedrate as tunable machining parameters. If off-time was selected as a tunable machining parameter, the $R_{a b}$ cannot be effectively controlled and once the curial machining situation occurred, the wire breaking will not be suppressed promptly. In our experiment, the workpiece and electrode were as follows: (1) workpiece (anode)-SKD11 alloy steels, (2) electrode (cathode), $\phi 0.25$-mm brass wire.

\subsection{The sparking monitoring system of WEDM process}

In a WEDM control system, a process-dependent parameter, called a sensing parameter, must be detected to monitor the gap state and to improve the machining performance. Usually, an average gap voltage was used to keep a small distance between the electrode and the workpiece in most commercial EDM machines. We can zoom in the time evolution of the gap voltage and the corresponding current as shown in Fig. 1. There are three types of discharges, normal pulse, arc pulse, and short-circuit pulse. In order to improve the machining efficiency and stability, it is necessary to develop an on-line monitor to classify these pulses.

According to the period of the high voltage sustained before sparking, i.e., the ignition delay time, the normal pulse has a relatively longer ignition delay time than the others. From this observation, a WEDM pulse classification and monitoring system has been developed. Because of the rather noisy character of the WEDM process, the voltage level between arc pulse and short-circuit pulse is difficult to be differentiated from each other. Hence, without losing too much information for control purpose, the short-circuit pulse and arc pulse are categorized in the same pulse group. Accordingly, only two types of pulse, normal pulse and abnormal pulse (including arc pulse and short-circuit pulse), are discriminated in our sparking monitoring system. Details of the discriminating principles can be found in our previous work [10]. The AX5216 card in the PC was used to count the number of pulses including normal pulses and abnormal pulses. The ratio of the counted number of the abnormal pulses to that of total pulses in a selected sampling period is defined as the abnormal ratio $R_{a b}$. In our system the sampling period ranges from 2 to $65,535 \mathrm{~ms}$.

\section{Self-tuning fuzzy inferences and grey prediction control system}

3.1 The controlled variable and its reference for the WEDM control system

According to the previous studies [10] associated with wire breaking, the abnormal ratio $R_{a b}$ is correlated with the gap state in the WEDM process. Thus it is taken as a controlled variable for evaluating the control performance in our control system. In this section, effects of the tunable machining parameters on the controlled variable will be investigated, which can be used to aid the design of the fuzzy controller. Figure 2 shows the effect of arc off-time on the feedrate and the abnormal ratio $R_{a b}$ when the servo reference voltage is $35 \mathrm{~V}$. When the arc off-time is set to a large value, the feedrate and the abnormal ratio $R_{a b}$ are all small. Such less-inefficient machining is due to the more conservative machining parameters. By stepwise reducing the arc off-time, the machining speed can be improved, but the wire rupture will take place eventually. The effect of

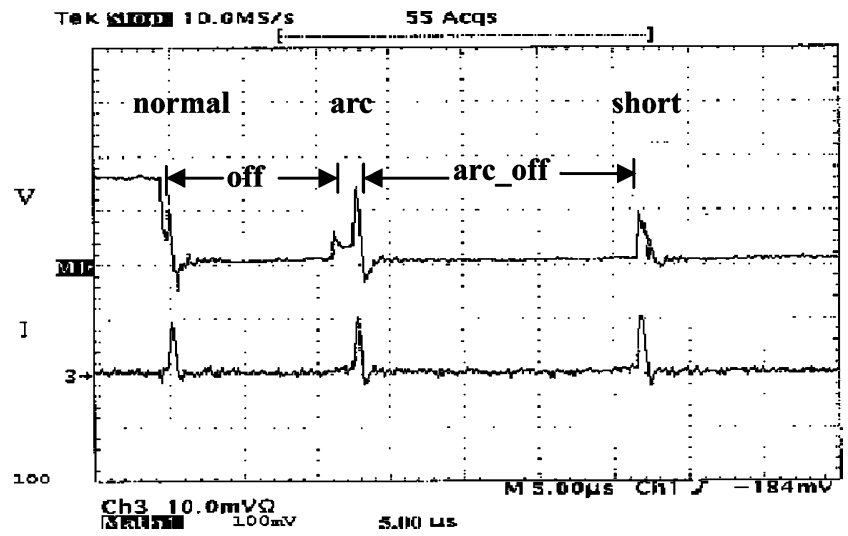

Fig. 1 Gap state: gap voltage and current waveforms of a WEDM process 


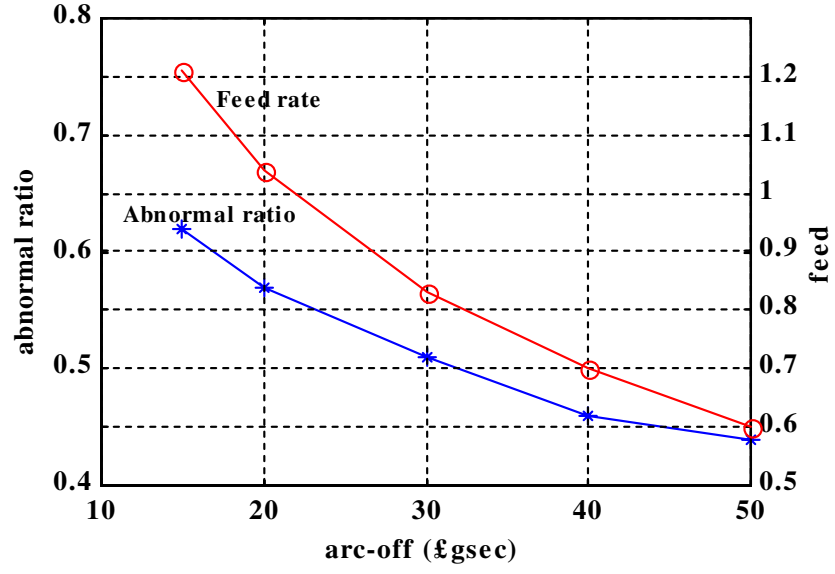

Fig. 2 Effect of the arc off-time on feedrate and abnormal ratio

feedrate on the $R_{a b}$ is given in Fig. 3. Similarly, by stepwise increasing the feedrate, the machining speed apparently increases. But the wire does not break down until $R_{a b}$ is beyond the safety level. In summary, the two tunable machining parameters are highly correlated with the $R_{a b}$ and the machining speed. In other words, the desired setting of the $R_{a b}$ can be achieved by concurrently tuning the arc off-time and the feedrate.

However, the higher the machining speed, the more likely the wire rupture would occur. The optimal $\left(R_{a b}\right)_{r e f}$ is a compromise between the ultimate machining speed and the occurrence of wire breaking. According to the results of influences of the machining parameters on machining characteristics $[12,13]$, in addition to the workpiece height, the abnormal ratio $R_{a b}$ can be tuned mainly by machining parameters, such as feedrate (or the reference servo voltage), the on-time and arc off-time. The optimal level of $\left(R_{a b}\right)_{r e f}$ can be obtained as follows: firstly, the workpiece height and on-time are set, and then by stepwise increasing the feedrate and decreasing the arc off-time, the optimal level of $\left(R_{a b}\right)_{\text {ref }}$ with faster machining speed but no wire breaking was determined. Through several experiments, the average value

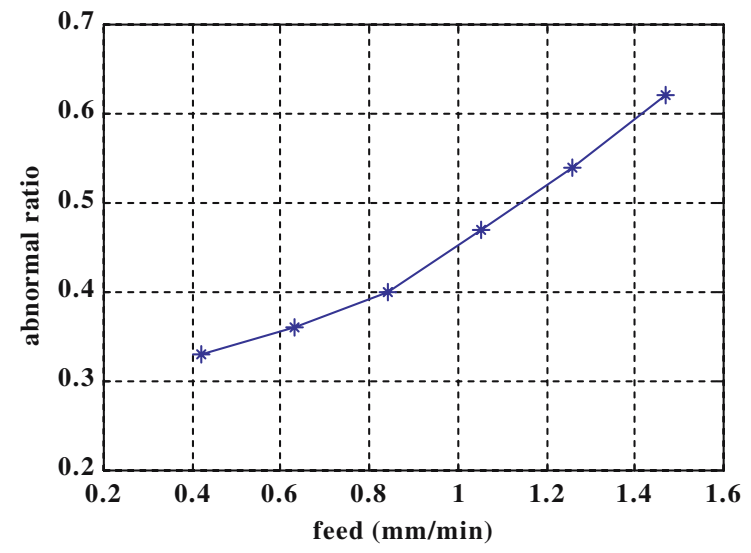

Fig. 3 Effect of the feedrate on abnormal ratio

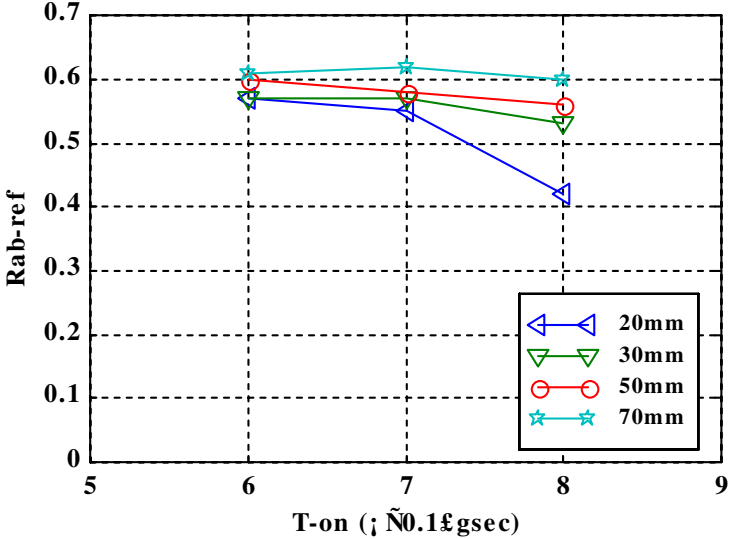

Fig. 4 Effect of the on-time on the $\left(\mathrm{R}_{\mathrm{ab}}\right)_{\text {ref }}$ in different thickness of workpiece

of $\left(R_{a b}\right)_{\text {ref }}$ was shown in Fig. 4. It was found from our experiments that the optimal level of $\left(R_{a b}\right)_{\text {ref }}$ has slight variation with other machining parameters. Figure 4 shows that the variation of its value is small while the cutting thickness is larger than $20 \mathrm{~mm}$. Accordingly, the setting for the optimal $\left(R_{a b}\right)_{r e f}$ can be ranged from 55 to $60 \%$ in the case of the cutting thickness being larger than $20 \mathrm{~mm}$. It is suggested to use a larger reference value of $60 \%$ in the case of unchangeable or fixed thickness workpiece. As for the case of a changeable thickness workpiece, the lower one, for example $55 \%$, is often advised.

\subsection{Compensation of time-delayed signal by grey predictor}

In Fig. 5, there is an obvious oscillation in the $R_{a b}$ that is measured in real-time. It is denoted by the original data, which possesses many high-frequency components. In order to attenuate the fluctuation of the original $R_{a b}$, a low-pass filter is usually employed to disclose the trend of the evolution of the gap state. The filtered data is also shown in Fig. 5, in which the details to determine the cutoff frequency of the low-pass filter can be seen in [9]. Compared to the evolution of the original signal, the filtered signal has an obvious time-delayed phenomenon, which will increase the risk of wire rupture, especially under the high-speed machining condition.

In order to cope with the time-delayed problem, a grey predictor [11] is used to compensate the filtered data. Based on the theory of grey prediction and associated data processing, the $p$-step compensated value of $\mathrm{y}^{(0)}(0)$, through grey model GM $(1,1)$, can be calculated by $[14]$

$\widehat{y}^{(0)}(k+p)=\left(1-e^{c}\right)\left[y^{(0)}(0)-\frac{b}{c}\right] e^{-c(k+p)}$

Where $p$ is the compensated step size, $\mathrm{y}^{(0)}$ is a filtered data sequence, $c$ and $b$ are the development coefficient and the grey input, respectively [11]. 


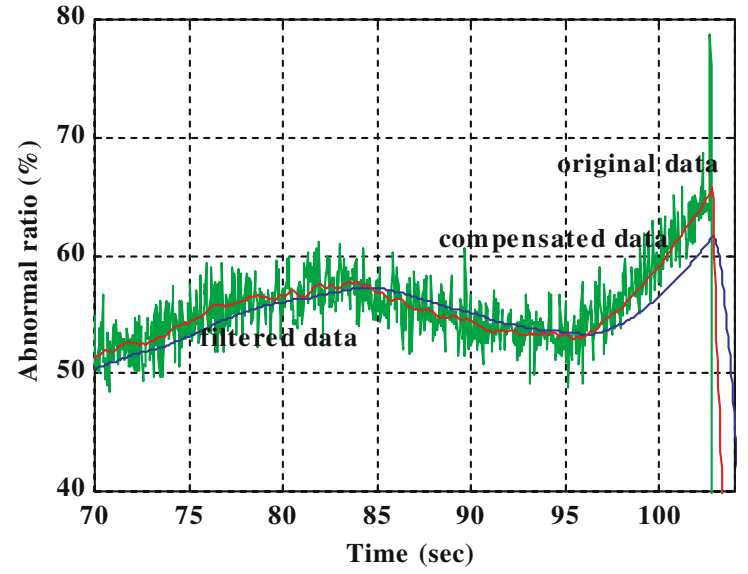

Fig. 5 The original, filtered and compensated $R_{a b}$ data

From the results in [9], the cut-off frequency of the second-order low-pass digital Butterworth filter is 0.012 times one half of sampling frequency of the original data, and the compensated step size of the grey predictor is 37 steps and these parameters are adopted in this paper. The compensated data, shown in Fig. 5, has good synchronism with the progression of the original signal.

\subsection{Gain self-tuning fuzzy controller}

In order to improve the machining efficiency under different machining conditions, a gain self-tuning fuzzy controller is used. Figure 6 is the configuration of the selftuning fuzzy inference with grey prediction control system, in which the $R_{a b}$ is taken as controlled variable to represent the gap state. In order to keep the $R_{a b}$ at a pre-defined level, the inputs to the controller including the error $e$ and the change of error $c e$, are described as follows:

$e(k)=\left(R_{a b}\right)_{r e f}-\left(R_{a b}\right)_{p}(k)$

$c e(k)=\left(R_{a b}\right)_{p}(k-1)-\left(R_{a b}\right)_{p}(k)$

$E=e(k) \times G e$

$C E=c e(k) \times G c e$

$$
C E=c e(k) \times G c e
$$
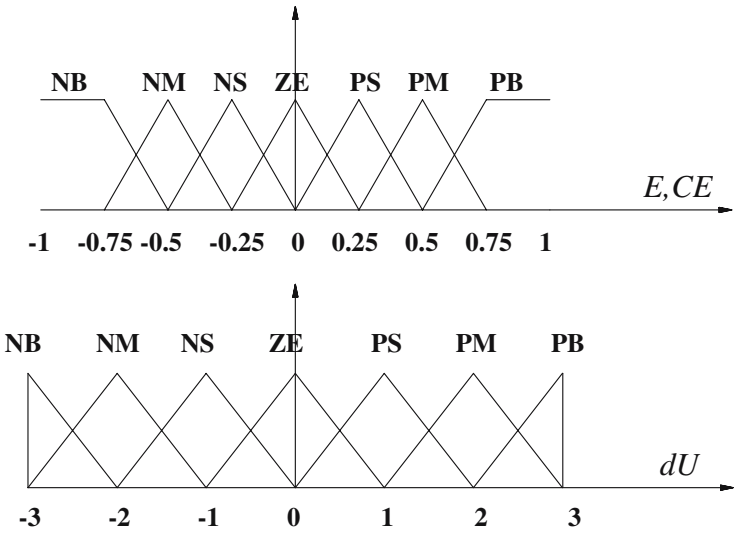

Fig. 7 Membership function of a $E, C E ; \mathbf{b} d U$

Where $\left(R_{a b}\right)_{r e f}$ is the reference for the abnormal ratio, $\left(R_{a b}\right)_{f}$ is the abnormal ratio processed by the low-pass filter from the original $R_{a b}$, and $\left(R_{a b}\right)_{p}$ is the abnormal ratio compensated by the grey predictor from the $\left(R_{a b}\right)_{f}$ Ge and Gce are input scaling factors and in our cases their values are $G e=5$ and $G c e=40$, respectively.

$E$ and $C E$ in Eq. $(2 \mathrm{a}, \mathrm{b})$ are corresponding fuzzy variables. Each fuzzy variable is quantified into seven term sets: NB-Negative Big, NM-Negative Medium, NS-Negative Small, ZE-Zero, PS-Positive Small, PM-Positive Medium, and PB-Positive Big. The triangular membership functions for each fuzzy term set and the universes of discourse for each fuzzy variable, including input and output variable, are shown in Fig. 7.

As Lee [15] pointed out, the rule bases of fuzzy inference can be derived from a dynamic system response. A typical response of the $R_{a b}$ in WEDM process is shown in Fig. 8, where the dash line denotes the pre-defined reference. At the point $a$, the $R_{a b}$ error, $E$, is $\mathrm{PB}$ and the change of the $R_{a b}, C E$, is ZE, which means that the current gap state is very secure, or more conservative, and moreover remains unchanged. Hence the change of control action $d U$, the arc off-time, should be significantly reduced to improve the machining speed. This linguistic control strategy can be described in fuzzy inference, as

IF $E$ is $P B$ and $C E$ is $Z E$, THEN $d U$ is $N B$

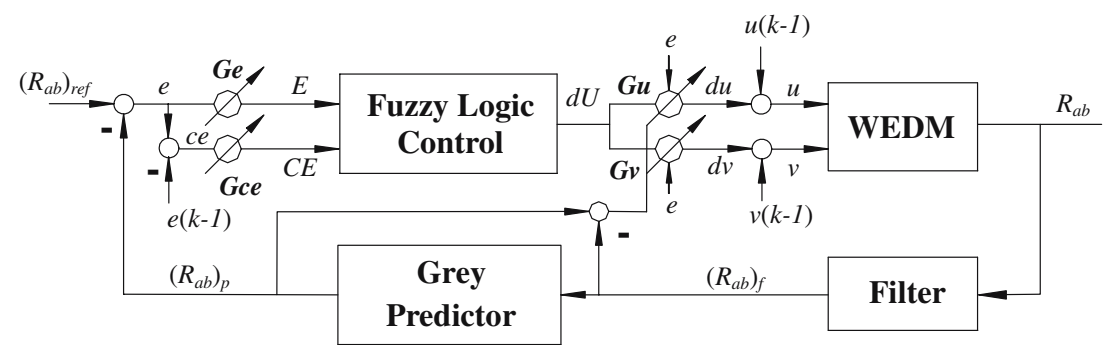

Fig. 6 Self-tuning fuzzy logic and grey prediction control system 


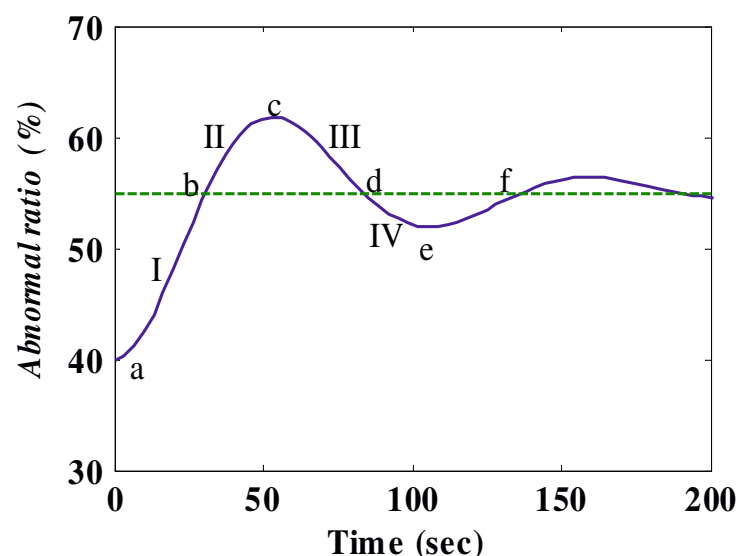

Fig. 8 A typical example of the $R_{a b}$ response for WEDM processes

In the same way, the other points such as $b, c, d \ldots$ can be described and the rule bases are summarized in Table 2. In addition, from Figs. 2 and 3 , the effect on the $R_{a b}$ by adjusting arc off-time is negatively correlated with that by the feed rate. By applying the inversion of the results of fuzzy inference to setting about the feedrate, the rule bases for arc off-time can be directly applied to those for the feedrate without any modification. As a result, the design procedure of the controller can be greatly simplified.

According to the height defuzzification method [16], the crisp output of fuzzy inference $d U$ can be determined. The associated increment control actions in Eq. $(4 a, b)$ with arc off-time and the feedrate can be calculated from $d U$ by multiplying the scaling factors, $G u$ and $G v$, respectively. The actual control actions at the $k$ th sampling instant are calculated by

$u(k)=u(k-1)+d u(k)$

$v(k)=v(k-1)+d v(k)$

where

$d u(k)=d U(k) \times G u$

$d v(k)=d U(k) \times G v$
$G u=K u|e| \Phi(\alpha, E, C E) \Psi\left(o l, d_{p f}\right)$

$G v=K v|e| \Phi(\alpha, E, C E) \Psi\left(o l, d_{p f}\right)$

$\Phi(\alpha, E, C E)=g 1+g 2 \times f(C E, 5 * E)$

$\Psi\left(o l, d_{p f}\right)=o l\left(1+100.0 * d_{p f}\right) \quad e<0.01$

$u(k), u(k-1) \quad \begin{gathered}\text { Arc off-time at the } k \text { th },(k-1) \text { th sampling } \\ \text { instant respectively }\end{gathered}$
$v(k), v(k-1) \quad$ The inversion of feedrate(i.e.1/feedrate) at the $k$ th, $(k-1)$ th sampling instant respectively

$d u(k) \quad$ Increment of arc off-time at the $k$ th sampling instant $d v(k) \quad$ Increment of the inversion of feedrate at the $k$ th sampling instant

$d U(k) \quad$ Crisp output of fuzzy inference at the $k$ th sampling instant

$\mathrm{Gu} \quad$ Output-scaling factor for the arc off-time

Gv Output-scaling factor for the inversion of feedrate

$g 1=0.5 \times(1.0+\alpha)$

$g 2=0.5 \times(1.0-\alpha)$

$f(x, \alpha)=\frac{1-\exp (-\alpha * x)}{1+\exp (-\alpha * x)}:$ hyperbolic tangent function

$d_{p f}=\left(R_{a b}\right)_{p}-\left(R_{a b}\right)_{f}$

\begin{tabular}{ll}
\hline$K u, K v$ & The global gain about control actions \\
$\alpha$ & I,III region tunable parameter \\
$o l$ & Overloading parameter \\
\hline
\end{tabular}

sampling time $\Delta t=40 \mathrm{~ms}$

Each of the tunable output-scaling factors, $\mathrm{Gu}$ and $\mathrm{Gv}$, has three parts: (1) The $R_{a b}$ error, $|e|$, is called the

Table 2 Rule bases of the FLC

\begin{tabular}{lllllll}
\hline$C E \mid E$ & NB & NM & NS & ZE & PS & PM \\
\hline PB & ZE & NS & NM & NB & NB & NB \\
PM & PS & ZE & NS & NM & NB & NB \\
PS & PM & PS & ZE & NS & NM & NB \\
ZE & PB & PM & PS & ZE & NS & NM \\
NS & PB & PB & PM & PS & ZE \\
NM & PB & PB & PB & PM & PS & NM \\
NB & PB & PB & PB & PM & NS \\
& & & & & PE
\end{tabular}


attraction-causing factor. Its function is to attract the $R_{a b}$ to the set-point, $\left(R_{a b}\right)_{r e f}$. (2) The overshoot-attenuating factor $\Phi(\alpha, E, C E)$ is to attenuate impending overshoot or undershoot by reducing control actions in regions I and III (referring to Fig. 8). Instead of tuning the massive rule bases [17], this factor $\Phi(\alpha, E, C E)$ in Eq. (6), is synthesized by a hyperbolic tangent function given in Eq. (8). Since a few parameters would be tuned, the task of design can be apparently simplified. The smaller the parameter $\alpha$ is and the more the level to be attenuated. But too small a value will reduce the system response. (3) The overloading factor $\Psi\left(o l, d_{p f}\right)$ is to suppress the overloading phenomenon by enhancing the control actions once the $R_{a b}$ apparently exceeds the safety level. For example, at the instant when the cutting thickness is increasing, the $d_{p f}$ in the $\Psi$ will increase drastically and accordingly enhance control actions to prevent the wire from breaking. Effect of $K u$ and $K v$ is similar to the gain of a general controller where a larger gain results in faster but unstable response.

\section{Experimental result and discussion}

Improving the cutting speed of machining a workpiece with changeable thickness is experimentally verified in this section. For the purpose of comparison, three methods are tested - (1) the constant $\left(R_{a b}\right)_{\text {ref, }}$ (2) the higher constant but temporarilyreduced $\left(R_{a b}\right)_{\text {ref }}$ according to the machining condition, and (3) the conventional constant servo reference voltage control, i.e. keeping the arc off-time and the servo reference voltage constant in the entire machining. In the experiments, the selftuning fuzzy control with grey prediction is implemented in the PC using C language. A RS485 interface card installed in the $\mathrm{PC}$ is used for the data communication between the $\mathrm{PC}$ and the machine. The sampling time used in the experiments is $40 \mathrm{~ms}$. Table 3 gives the corresponding machining parameters initially set, and Fig. 9 depicts the cross section of workpiece used. Table 4 shows the parameters of the controller in our experiments.

Before discussing the experimental results, effects of the changeable cutting thickness on the machining gap state are described as follows: The energy principle shown in Eq. (9) points out that the potential difference $V$ between two points equals the product of the electric intensity $E$ and each other's separation $D$. In the case of the WEDM process, the separation $D$ can be considered as the gap size $g(t)$ between a workpiece and the wire. It can be obtained from Eq. (10), in which $V$ is associated with the voltage of the power and $E$ is the electric field intensity in the gap. During machining, the moving wire electrode gradually approaches the workpiece, i.e., the gap becomes narrow, and the electrical intensity $E$ within the gap will gradually increase. Not until it is over the critical electric intensity $E_{C}$ of the dielectric fluid will the spark take place. The gap size at that instant is denoted by the sparking gap size $g_{S}$, as shown in Eq. (11). When the voltage of the power is raised, the gap size at that instant, $g_{L}$, largely dictates which events are most likely to take place at the gap. Specifically, if the $g_{L}$ is wider than the $g_{S}$, the ignition delay time $t_{d}$ will be larger than zero and then the possibility of generating normal discharge is large; otherwise the abnormal pulse will appear if the $g_{L}$ is near to the $g_{S}$ or smaller. In summary, Eq. (12) shows this relation, which points out that the gap size dominantly affect the type of pulse to be generated, or the gap state.

$V=E \times D$

$g(t)=V / E$

$g_{s}=V / E_{c}$

$\left\{\begin{array}{l}g_{L}>g_{s} \Rightarrow t_{d}>0 \Rightarrow \text { Normal discharge } \\ g_{L} \leq g_{s} \Rightarrow t_{d}=0 \Rightarrow \text { Abnormal discharge }\end{array}\right.$

During the steady machining state, the erosion rate $E_{r}$ $\left(\mathrm{mm}^{2} / \mathrm{min}\right)$ is dynamically equilibrium with the feedrate of machine table, $F_{r}(\mathrm{~mm} / \mathrm{min})$ and then the gap size can be approximately considered as constant. If the machining condition alters, the change of the gap size is related with the erosion rate $E_{v}$, the feedrate of machine table $F_{r}$ and the thickness of the workpiece $h$. It is described in Eq. (13). It shows that the change of the cutting thickness will affect the gap size, or the gap state, which will be seen in the following experiments.

$\dot{g}=\frac{E_{r}\left(T_{o n}, A_{o n}, \text { Freq }\right)}{h}-F_{r}$

Now let us return to the subject of this section and discuss the experimental results of the three methods in this study.

\subsection{Method 1- the constant reference for the $\boldsymbol{R}_{a b}$}

At the instant when the moving electrode reached to the step change in thickness shown in Fig. 9, the change of gap size would be negative. It is due to the suddenly increasing thickness which causes a decrease to the first term in the right hand side of Eq. (13). Figure 10a shows that this narrow gap size brings about a lot of abnormal discharges. In Fig. $10 \mathrm{a}$, the fluctuating signal of $R_{a b}$ indicates the original on-line measured $R_{a b}$, while the solid line stands for the filtered and compensated one. Because the increas- 
Table 3 Machining parameters

\begin{tabular}{llllllllll}
\hline Method & & & Arc & Arc & Servo & Feed & Wire & Wire & Flushing \\
\hline No. & On-time & Off-time & On-time & Off-time & Voltage & Rate & Speed & Tension & Pressure \\
\hline 1 & 6 & 15 & 4 & 30 & - & 1.60 & 4 & 10 \\
2 & 6 & 15 & 4 & 16 & - & 2.20 & 4 & 10 \\
3 & 6 & 15 & 4 & 29 & 63 & - & 4 & 2 \\
\hline
\end{tabular}

ing $R_{a b}$ exceeds the safety level, the wire electrode is burn out very soon. Machining parameters are listed in Table 3 (method no. 1). Clearly, the larger the change of workpiece thickness, the lower the safety level of $R_{a b}$ should be set to complete a whole machining process. But too low a reference level of $R_{a b}$ will sacrifice the cutting speed. Taking these into consideration, it is found from our experiments that the proper level of $R_{a b}$ for the $20-\mathrm{mm}$ step increase in the workpiece thickness is $42 \%$. Figures $10 \mathrm{~b}$ and $\mathrm{c}$ show the time evolution of the controlled $R_{a b}$ and the corresponding control actions, respectively under the same machining condition as that of Fig. 10a. The associated parameters of the controller are given in Table 4 (method no.1). Viewing from these figures, as soon as the traveling wire electrode contacts the step change of thickness and then the $R_{a b}$ dramatically raises, the control action is taken to immediately cope with the crucial machining condition. The fast increasing $R_{a b}$ can be effectively and promptly suppressed through reducing the feedrate and elongating the arc off-time by the controller. There was a slight decline in the $R_{a b}$ as about $300 \mathrm{~s}$ have escaped (see Fig. 10b), i.e. when the traveling electrode has moved away from the location of the changing thickness. The arc off-time and the feedrate are gradually tuned back by the controller to keep the $R_{a b}$ around the pre-defined level, and to improve the cutting speed.

\subsection{Method 2-the adaptive reference for the $\boldsymbol{R}_{a b}$}

To prevent the wire electrode from breaking as the cutting thickness is suddenly changing, the value of the $\left(R_{a b}\right)_{\text {ref }}$ is set to be $42 \%$ in previous section. In fact, as stated in the section 3.1, when the cutting thickness is larger than $20 \mathrm{~mm}$, the optimal $\left(R_{a b}\right)_{\text {ref }}$ may be set to $55 \%$. But under such a high machining speed, whatever the design of the controller was, the wire rupture always occurred as the

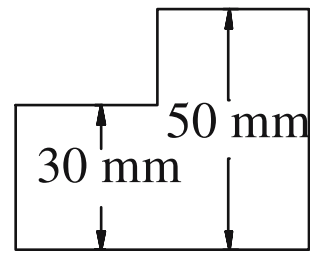

Fig. 9 The cross section of the workpiece cutting thickness was increasing. In order to overcome this difficulty and keep the machining at optimal level, temporary reduction of the optimal $\left(R_{a b}\right)_{\text {ref }}$ may be a feasible strategy.

Figure 11a shows the same phenomenon as in Fig. 10a but it endured a shorter period before the wire rupture. It is due to the fast cutting speed resulted from the higher $\left(R_{a b}\right)_{r e f}, 55 \%$, in this case and the associated machining parameters are listed in Table 3 (method no. 2). The $\left(R_{a b}\right)_{\text {ref }}$ shown in Fig. $11 \mathrm{~b}$ can be determined as follows. It is initially set to be $55 \%$ to maintain an optimal machining speed. As the electrode reaches the step change of thickness, the temporary reduction of the $\left(R_{a b}\right)_{\text {ref }}$ is set to suppress the geometrical discontinuity-induced instability. The amount of the reduction in the $\left(R_{a b}\right)_{r e f}$ depends on the size of sudden change in thickness. As mentioned in section 4.1 , the temporarily-reduced $\left(R_{a b}\right)_{\text {ref }}$ is taken as $42 \%$. In addition, the time when the $\left(R_{a b}\right)_{\text {ref }}$ should be reduced is critical for the machining process. Specifically, if it is too late, the wire ruptures may occur. The signal erp, $\left(R_{a b}\right)_{p}-\left(R_{a b}\right)_{f}$, will be taken to detect and trigger the time for reducing the $\left(R_{a b}\right)_{\text {ref }}$. In our experiment, as soon as the signal erp is greater 0.015 shown in Fig. $11 \mathrm{~b}$, the $\left(R_{a b}\right)_{r e f}$ is immediately set to $42 \%$. After the electrode cuts into the workpiece about $1 \mathrm{~mm}$, the adaptive $\left(R_{a b}\right)_{\text {ref }}$ is gradually tuned back to $55 \%$ to keep an optimal machining speed. Figure $11 \mathrm{~b}$ shows the resulted time evolution of the controlled $R_{a b}$, which can be successfully controlled to follow the adaptive $\left(R_{a b}\right)_{\text {ref }}$ to keep an optimal machining. Figure 11c gives the corresponding control actions provided by the developed fuzzy controller. By comparing Fig. 11c with Fig. 10c, it can be seen that the time for the wire reaching the step change thickness in Fig. 11c is earlier due to the fast machining speed in method 2; accordingly, there are more adjustments in the tuned machining parameters to cope with this more crucial machining condition. After passing the geometric discontinuity in thickness, they are obviously tuned to improve the machining speed according to the adaptive $\left(R_{a b}\right)_{r e f}$.

\subsection{Method 3-the constant machining parameters}

For comparison purposes, the fuzzy controller is not involved here. In other words, the whole machining process 
Table 4 The parameters for the controller

\begin{tabular}{llllllll}
\hline Method no. & Ge & Gce & $\dot{a}$ & ol & $\mathrm{Ku}$ & $\mathrm{Kv}$ & $\left(R_{a b}\right)_{\text {ref }}$ \\
\hline 1 & 5 & 40 & 0.7 & 3.0 & 0.08 & 0.008 & 0.42 \\
2 & 5 & 40 & 0.7 & 3.0 & 0.08 & 0.008 & $0.55-0.42$ \\
3 & - & - & - & - & - & - & - \\
\hline
\end{tabular}

is under the conventional servo gap voltage control and the associated machining parameters were predefined. But in this case the wire rupture often occurred as the cutting thickness was increasing. From our experiments the allowable machining parameters are 63 volts in the servo reference voltage and $29 \mu \mathrm{s}$ in the arc off-time that have

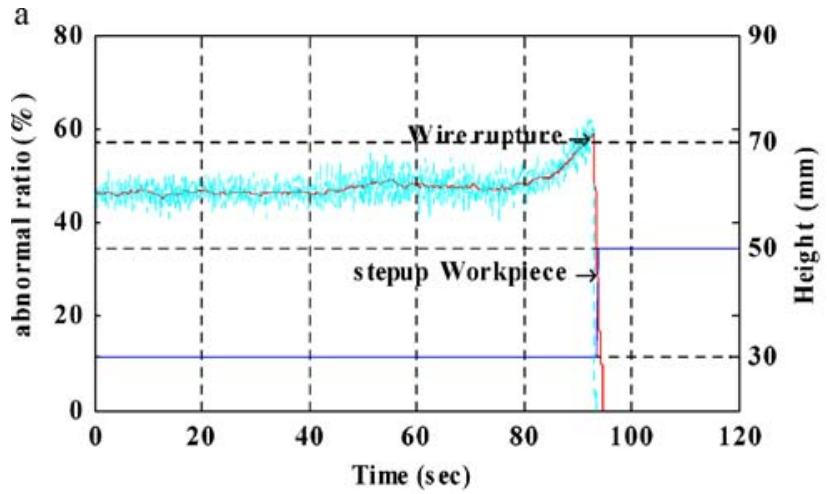

b
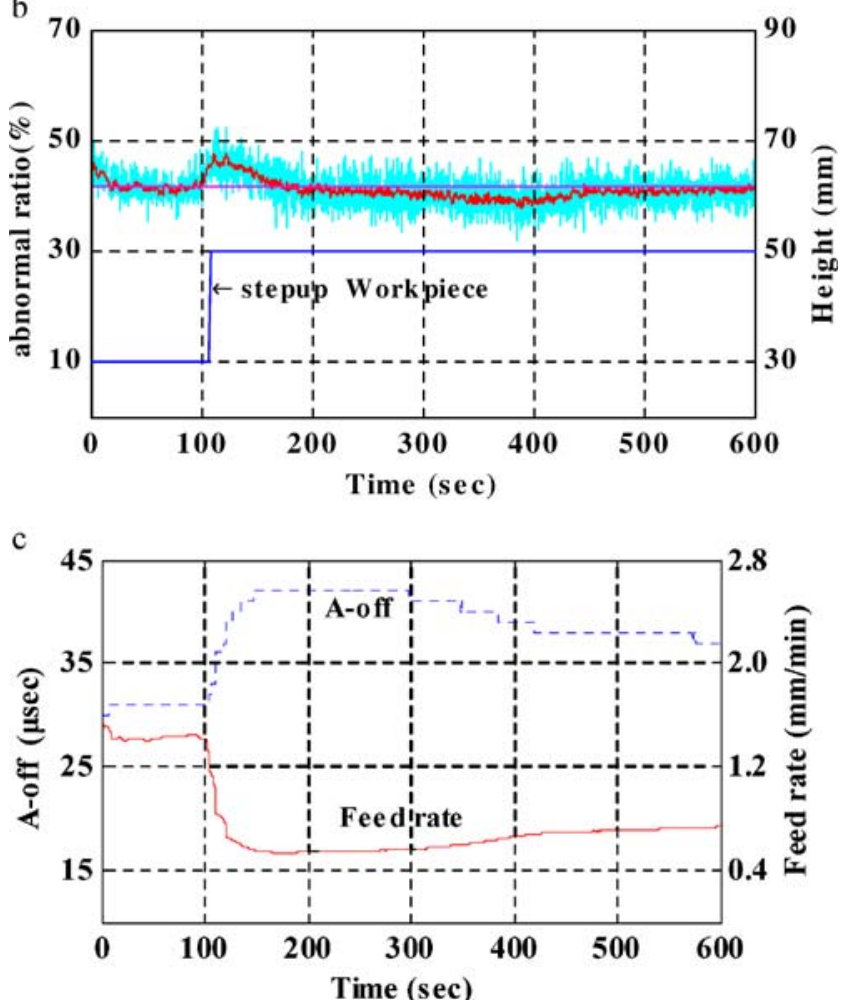

Fig. 10 Time evolution of a the $R_{a b}$ in the machining condition shown in Table 4. (method 1) - a wire rupture happened, $\mathbf{b}$ the $R_{a b}$ under the control of the method 1 and $\mathbf{c}$ control actions: the arc off-time and the feedrate
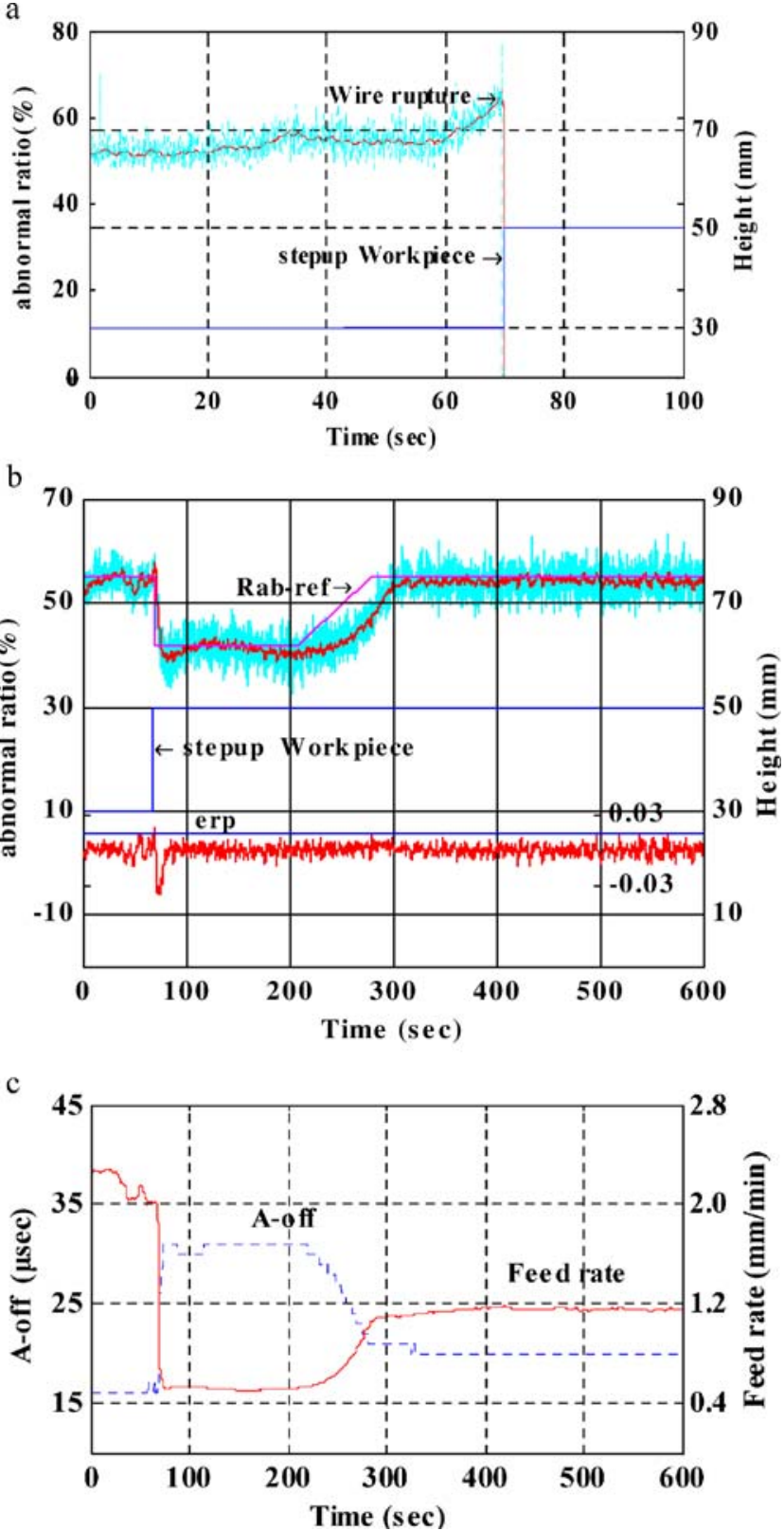

Fig. 11 Time evolution of a the $R_{a b}$ in the machining condition shown in Table 4 (method 2) - a wire rupture occurred; b the $R_{a b}$ under the control of the method 2 and $\mathbf{c}$ control actions: the arc off-time and the feedrate

rather fast machining speed and no wire breaking. The associated machining parameters in this case are listed in Table 3 (method no.3). Figure 12 shows the resulted time evolution of the $R_{a b}$. Because of the more conservative machining conditions, there is a slight increase in the $R_{a b}$ as the cutting thickness is increasing. There is a small peak before the slight increase of the $R_{a b}$. It is due to the interaction between the flush of the dielectric fluid and the sudden increasing thickness. At that instant the electrode has not yet contacted the wall of the upper part of the workpiece. 


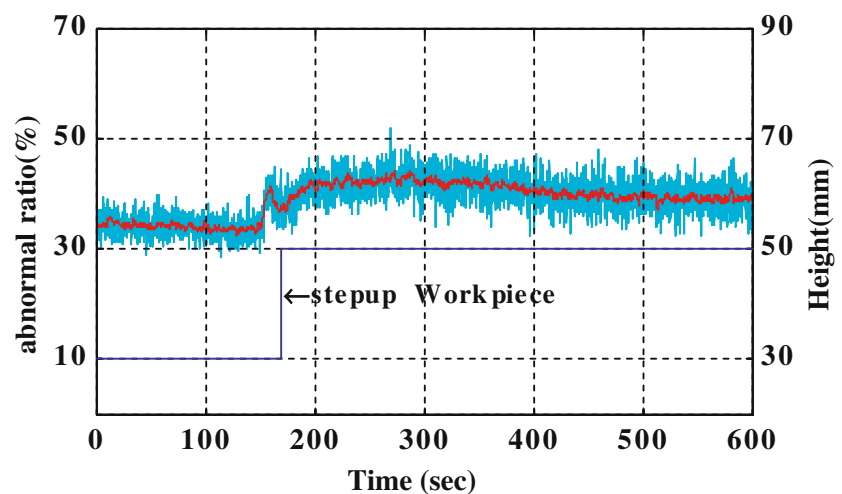

Fig. 12 Time evolution of the $R_{a b}$ in the machining condition shown in Table 4.(method 3)

Finally, the experimental results of the cutting speed for each method are summarized in Table 5. Because the predefined machining parameters in this experiment, such as on-time and flushing pressure etc., are set to be moderate, the maximum cutting speed in Table 5 only reaches $56.7 \mathrm{~mm}^{2} / \mathrm{min}$. Its value should be increased by further tuning up the associated machining parameters. Nevertheless, the relative performances for each method can be described in the following. One reason for the less efficiency in method 3 is due to the conservative machining parameters which are taken to prevent wire from breaking. Another is that the fuzzy controller is not adopted to tune the machining parameters in real-time and they remain unchanged in the entire machining process. The constant $\left(R_{a b}\right)_{r e f}$ according to the worst cutting condition is adopted in the method 1. Instead of the constant machining parameters, both the feedrate and the arc off-time are simultaneously tuned to keep the $R_{a b}$ at the constant level. Hence the machining speed in the method 1 is faster than that in method 3. As for the method 2, the temporarilyreduced $\left(R_{a b}\right)_{\text {ref }}$ is adopted according to the changing thickness, hence the higher $\left(R_{a b}\right)_{\text {ref }}$ can be specified to improve the machining efficiency. Thus the resulted cutting speed can be obviously improved for unmanned machining a workpiece with varying thickness.

Table 5 The comparison of the cutting speed

\begin{tabular}{lll}
\hline $\begin{array}{l}\text { Method } \\
\text { no. }\end{array}$ & $\begin{array}{l}\text { Machining area }\left(\mathrm{mm}^{2}\right) / \text { cutting speed } \\
\left(\mathrm{mm}^{2} / \mathrm{min}\right)\end{array}$ & Improvement \\
\hline 1 & $371.1 / 37.1$ & $27 \%$ \\
2 & $566.9 / 56.7$ & $94 \%$ \\
3 & $292.5 / 29.3$ & - \\
\hline
\end{tabular}

Note: the time of the machining: $10 \mathrm{~min}$

\section{Conclusion}

In this paper, experimental results on $\left(R_{a b}\right)_{r e f}$ show that the variance of the optimal setting for different cutting thickness is small when the cutting thickness is larger than $20 \mathrm{~mm}$. In these cases, the optimal $\left(R_{a b}\right)_{r e f}$ can be set constant as $55 \%$ in machining a workpiece with varying thickness. To cut a workpiece with varying thickness under such a fast machining condition, an algorithm for designing the $\left(R_{a b}\right)_{r e f}$ is proposed to cope with the geometrical discontinuity of the cutting thickness. Furthermore, a gain self-tuning fuzzy controller was used. The output scaling factor of the fuzzy controller can be adaptively tuned in a wide variety of machining conditions. Experimental results show that not only can the transient situation be immediately suppressed as the cutting thickness is suddenly increasing, but a stable performance can also be achieved. By the concurrent adjustment of the arc off-time and the feedrate, provided by the developed controller, the $R_{a b}$ can be successfully controlled to follow the adaptive $\left(R_{a b}\right)_{\text {ref }}$ at the optimal level, i.e. at the maximum cutting speed, when cutting a workpiece with varying thickness. Experimental results demonstrated that the proposed control strategy can obviously improve the cutting speed for unmanned machining a workpiece with varying thickness in the WEDM process.

\section{References}

1. Benedict GF (1987) Nontraditional manufacturing processes. Marcel Dekker, Inc.

2. Kinoshita N, Fukui M, Shichida H, Gamo G (1976) Study on E.D. M. with wire electrode; gap phenomena. Annals of the CIRP 25 (1):141-145

3. Tanimura T, Heuvelman CJ (1977) The properties of the servo gap sensor with sparking-erosion machining. Annals of the CIRP 26 (1):59-63

4. Huang YH, Zhao GG, Zhang ZR, Yu CY (1986) The identification and its means of servo feed adaptive control system in WEDM. Annals of the CIRP 35(1):121-123

5. Zhang W, Bai JC, Liu JC (1989) The application of adaptive control in WEDM. Proceedings of the International Symposium for Electro-Machining, (ISEM-9) 1989:378-381

6. Rajurkar KP, Wang WM (1994) WEDM identification and adaptive control for variable-height components. Ann CIRP 43 (1):199-202

7. Yan MT, Liao YS (1998) Adaptive control of the WEDM process using the fuzzy control strategy. J Manuf Syst 17(4):263-273

8. Liao YS, Woo JC (2000) Design of a fuzzy controller for the adaptive control of WEDM process. Int J Mach Tools Manuf 40(15):2293-2307

9. Lee WM, Liao YS (2003) Self-tuning fuzzy control with grey prediction for wire rupture prevention in WEDM. Int J Adv Manuf Technol 22(7-8):481-490

10. Liao YS, Chiu YY, Yan MT (1997) Study of wire breaking process and monitoring of WEDM. Int J Mach Tools Manuf 37 (4):555-567

11. Deng JL (1989) Introduction to grey system. J Grey Syst 1:1-24 
12. Tsai TC (1999) Application of neural network and genetic algorithms in the machining-parameters optimization for WEDM. Master Thesis, Mechanical Engineering, National Taiwan University

13. Liao YS, Yu YP (2004) The energy aspect of material property in WEDM and its application. J Mater Process Technol 149:77-82

14. Wong CC, Liang WC, Feng HM, Chiang DA (1998) Grey prediction controller design. J Grey Syst 10(2):123-131
15. Lee CC (1990) Fuzzy logic in control systems: fuzzy logic controller ${ }^{-}$Part I. IEEE Trans on Systems Man and Cybernetics 20:404-418

16. Driankov D, Hellendoorn H, Reinfrank M (1996) An introduction to fuzzy control. Springer, Berlin Heidelberg New York

17. Mudi RK, Pal RP (1999) A robust self-tuning scheme for PI- and PD-type fuzzy controllers. IEEE Trans on Fuzzy Syst 7(1):2-16 